\title{
On the Zeros of a Polynomial
}

\author{
Mohammad Syed Pukhta \\ Division of Agricultural Engineering, Sher-e-Kashmir University of Agricultural Sciences \\ \& Technology of Kashmir, Srinagar, India \\ E-mail: mspukhta_67@yahoo.co.in
}

Received February 27, 2011; revised May 25, 2011; accepted June 3, 2011

\section{Abstract}

In this paper we consider the problem of finding the estimate of maximum number of zeros in a prescribed region and the results which we obtain generalizes and improves upon some well known results.

Keywords: Polynomial, Zeros, Complex Number, Prescribed Region

\section{Introduction}

Let $p(z)=\sum_{i=0}^{n} a_{i} z^{i}$ be a polynomial of degree $n$ such that

$$
a_{n} \geq a_{n-1} \geq \cdots \geq a_{1} \geq a_{0}>0
$$

then according to a well known result of Enstrom and Kakeya, the polynomial $p(z)$, does not vanish in $|z|>1$. concerning the number of zeros of the polynomial in the region $|z| \leq \frac{1}{2}$, the following result is due to Mohammad [1].

Theorem A. Let $p(z)=\sum_{i=0}^{n} a_{i} z^{i}$ be a polynomial of degree $n$ such that

$$
a_{n} \geq a_{n-1} \geq \cdots \geq a_{1} \geq a_{0}>0,
$$

then the number of zeros of $p(z)$ in $|z| \leq \frac{1}{2}$, does not exceed

$$
1+\frac{1}{\log 2} \log \frac{a_{n}}{a_{o}} .
$$

Dewan [2] generalized Theorem A to the polynomials with complex coefficients and obtained the following result.

Theorem B. If $p(z)=\sum_{i=0}^{n} a_{i} z^{i}$ is a polynomial of degree $n$ with complex coefficients such that $\left|\arg a_{i}-\beta\right| \leq \alpha \leq \frac{\pi}{2}, i=0,1,2, \cdots, n$ for some real $\beta$ and

$$
\left|a_{n}\right| \geq\left|a_{n-1}\right| \geq \cdots \geq\left|a_{1}\right| \geq\left|a_{0}\right|,
$$

then the number of zeros of $p(z)$ in $|z| \leq \frac{1}{2}$ does not exceed

$$
\frac{1}{\log 2} \log \frac{\left|a_{n}\right|(\cos \alpha+\sin \alpha+1)+2 \sin \alpha \sum_{i=0}^{n-1}\left|a_{i}\right|}{\left|a_{0}\right|} .
$$

Theorem C. Let $p(z)=\sum_{i=0}^{n} a_{i} z^{i}$ be a polynomial of degree $n$ with complex coefficients. If

$\operatorname{Re} a_{i}=\alpha_{i}, \operatorname{Im} a_{i}=\beta_{i}$, for $i=0,1, \cdots, n$ and $\alpha_{n} \geq \alpha_{n-1} \geq \cdots \geq \alpha_{1} \geq \alpha_{0}>0$, then the number of zeros of $p(z)$ in $|z| \leq \frac{1}{2}$ does not exceed

$$
1+\frac{1}{\log 2} \log \frac{\alpha_{n}+\sum_{i=0}^{n}\left|\beta_{i}\right|}{\left|a_{0}\right|} .
$$

In this paper we generalize Theorem B and Theorem C under less restrictive conditions on the coefficients, which also improve upon them. More precisely, we prove the following.

Theorem 1. Let $p(z)=\sum_{i=0}^{n} a_{i} z^{i}$ be a polynomial of degree $\mathrm{n}$ with complex coefficients, such that $\left|\arg a_{i}-\beta\right| \leq \alpha \leq \frac{\pi}{2}, i=0,1,2, \cdots, n$. for some real $\beta$ and

$$
\left|a_{n}\right| \geq\left|a_{n-1}\right| \geq \cdots \geq\left|a_{1}\right| \geq\left|a_{0}\right|>0
$$

then the number of zeros of $p(z)$ in $|z|<\delta$, does not exceed 


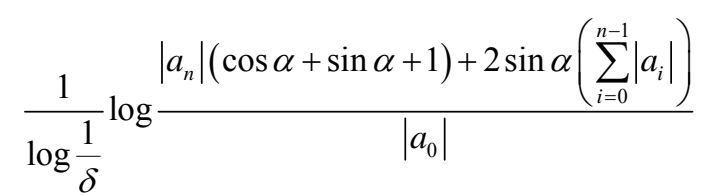

where $0<\delta<1$.

Theorem 2. Let $p(z)=\sum_{i=0}^{n} a_{i} z^{i}$ be a polynomial of degree $n$ with complex coefficients. If

$\operatorname{Re} a_{i}=\alpha_{i}, \operatorname{Im} a_{i}=\beta_{i}$, for $i=0,1, \cdots, n$ and $\alpha_{n} \geq \alpha_{n-1} \geq \cdots \geq \alpha_{1} \geq \alpha_{0}>0, \alpha_{n}>0$, then the number of zeros of $p(z)$ in $|z| \leq \delta, 0<\delta<1$ does not exceed

$$
1+\frac{1}{\log \frac{1}{\delta}} \log \frac{\alpha_{n}+\sum_{i=0}^{n}\left|\beta_{i}\right|}{\left|a_{0}\right|} \text {. }
$$

\section{Lemma}

We need the following lemma for proof of the theorems.

Lemma. Let $p(z)=\sum_{i=0}^{n} a_{i} z^{i}$ be a polynomial of degree $\mathrm{n}$ such that

$\left|\arg a_{i}-\beta\right| \leq \alpha \leq \frac{\pi}{2} ;\left|a_{i}\right| \geq\left|a_{i-1}\right|$ for some $i=0,1,2, \cdots, n$, then

$$
\left|a_{i}-a_{i-1}\right| \leq\left(\left|a_{i}\right|-\left|a_{i-1}\right|\right) \cos \alpha+\left(\left|a_{i}\right|+\left|a_{i-1}\right|\right) \sin \alpha .
$$

The proof of the above lemma is omitted as it follows from the lemma in [3].

\section{Proof of the Theorems}

Proof of Theorem 1. Consider the polynomial

$$
\begin{aligned}
g(z)= & (1-z) p(z)=(1-z)\left(a_{n} z^{n}+a_{n-1} z^{n-1}+\cdots+a_{1} z+a_{0}\right) \\
= & -a_{n} z^{n+1}+\left(a_{n}-a_{n-1}\right) z^{n} \\
& +\left(a_{n-1}-a_{n-2}\right) z^{n-1}+\cdots+\left(a_{1}-a_{0}\right) z+a_{0}
\end{aligned}
$$

For $|z| \leq 1$, we have

$$
\begin{aligned}
& g(z)=\left|a_{n}\right|+\sum_{i=1}^{n}\left|a_{i}-a_{i-1}\right|+\left|a_{0}\right| \\
& \leq\left|a_{n}\right|+\sum_{i=1}^{n}\left(\left|a_{i}\right|-\left|a_{i-1}\right|\right) \cos \alpha+\sum_{i=1}^{n}\left(\left|a_{i}\right|+\left|a_{i-1}\right|\right) \sin \alpha+\left|a_{0}\right|
\end{aligned}
$$

(by using Lemma)

$=\left|a_{n}\right|(\cos \alpha+\sin \alpha+1)+2\left(\sum_{i=0}^{n-1}\left|a_{i}\right|\right) \sin \alpha$

$-\left|a_{0}\right|(\cos \alpha+\sin \alpha-1)$

$\leq\left|a_{n}\right|(\cos \alpha+\sin \alpha+1)+2 \sin \alpha \sum_{i=0}^{n-1}\left|a_{i}\right|$.
If $f(z)$ is regular, $f(0) \neq 0$ and $f(z) \leq M$ in $|z| \leq 1$, then ([4], p.171) the number of zeros of $f(z)$ in $|z| \leq \delta, 0<\delta<1$ does not exceed $\frac{1}{\log \frac{1}{\delta}} \log \frac{M}{|f(0)|}$. Apply this result to $g(z)$ in $|z| \leq \delta$ does not exceed

$$
\frac{1}{\log \frac{1}{\delta}} \log \frac{\left|a_{n}\right|(\cos \alpha+\sin \alpha+1)+2 \sin \alpha\left(\sum_{i=0}^{n-1}\left|a_{i}\right|\right)}{\left|a_{0}\right|} .
$$

All the number of zeros of $p(z)$ in $|z| \leq \delta$ is also equal to the number of zeros of $g(z)$ in $|z| \leq \delta$. This completes proof of Theorem 1.

\section{Proof of Theorem 2.}

Consider

$$
g(z)=(1-z) p(z)=a_{n} z^{n+1}+\sum_{i=1}^{n}\left(a_{i}-a_{i-1}\right) z^{i}+a_{0}
$$

For $|z| \leq 1$,

$$
\begin{aligned}
|g(z)| & \leq\left|a_{n}\right|+\sum_{i=1}^{n}\left|a_{i}-a_{i-1}\right|+\left|a_{0}\right| \\
& \leq \alpha_{n}+\left|\beta_{n}\right|+\sum_{i=1}^{n}\left\{\left|\alpha_{i}-\alpha_{i-1}\right|+\left|\beta_{i}-\beta_{i-1}\right|\right\}+\alpha_{0}+\left|\beta_{0}\right| \\
& \leq \alpha_{n}+\left|\beta_{n}\right|+\sum_{i=1}^{n}\left(\alpha_{i}-\alpha_{i-1}\right)+\sum_{i=1}^{n}\left(\left|\beta_{i}\right|+\left|\beta_{i-1}\right|\right)+\left|\beta_{0}\right| \\
& =2\left(\alpha_{n}+\sum_{i=0}^{n}\left|\beta_{i}\right|\right)
\end{aligned}
$$

and using the same argument as in proof of Theorem 1, the proof of Theorem 2 follows.

Remark 1. For $\delta=\frac{1}{2}$, Theorem 1 is a refinement of Theorem B and for $\delta=\frac{1}{2}$, and $\alpha=\beta=0$, it gives a refinement of Theorem A.

Remark 2. Theorem $\mathrm{C}$ can be deduced as a particular case of Theorem 2 by putting $\delta=\frac{1}{2}$. If we put $\beta_{i}=0,0 \leq i \leq n$ in Theorem 2, we can deduce Theorem A.

Corollary 1. Let $p(z)=\sum_{i=0}^{n} a_{i} z^{i}$ be a polynomial of degree $n$, such that

$$
\alpha_{n} \geq \alpha_{n-1} \geq \cdots \geq \alpha_{1} \geq \alpha_{0},
$$

then the number of zeros of $p(z)$ in $|z| \leq \delta, 0<\delta<1$, does not exceed

$$
1+\frac{1}{\log \frac{1}{\delta}} \log \frac{\alpha_{n}}{\left|a_{0}\right|} .
$$




\section{Acknowledgements}

Author is highly thankful to the referees for their valuable suggestions.

\section{References}

[1] N. K. Govil and Q. I. Rehman, "On the Enstrom Kakeya Theorem," Tohoku Mathematical Journal, Vol. 20, No. 2, 1968, pp. 126-136. doi:10.2748/tmj/1178243172
[2] Q. G. Mohammad, "On the Zeros of the Polynomials," American Mathematical Monthly, Vol. 72, No. 6, 1965, pp. 631-633. doi:10.2307/2313853

[3] E. C. Titchmarsh, "The Theory of Functions," 2nd Edition, Oxford University Press, London, 1939.

[4] K. K. Dewan, "Extremal Properties and Coefficient Estimates for Polynomials with Restricted Zeros and on Location of Zeros of Polynomials," Ph.D Thesis, Indian Institutes of Technology, Delhi, 1980. 